Techniques \& Culture

$67 \mid 2017$

Low Tech ? Wild Tech!

\title{
Wild Tech
}

Repenser l'invention avec Charlie Bowers

\section{Emmanuel Grimaud}

\section{(2) OpenEdition \\ Journals}

Édition électronique

URL : https://journals.openedition.org/tc/8493

DOI : $10.4000 /$ tc. 8493

ISSN : 1952-420X

Éditeur

Éditions de l'EHESS

\section{Édition imprimée}

Date de publication : 6 juin 2017

Pagination : 258-279

ISBN : 978-2-7132-2707-3

ISSN : 0248-6016

Référence électronique

Emmanuel Grimaud, «Wild Tech », Techniques \& Culture [En ligne], 67 | 2017, mis en ligne le 06 juin 2019, consulté le 29 septembre 2022. URL : http://journals.openedition.org/tc/8493 ; DOI : https:// doi.org/10.4000/tc. 8493 


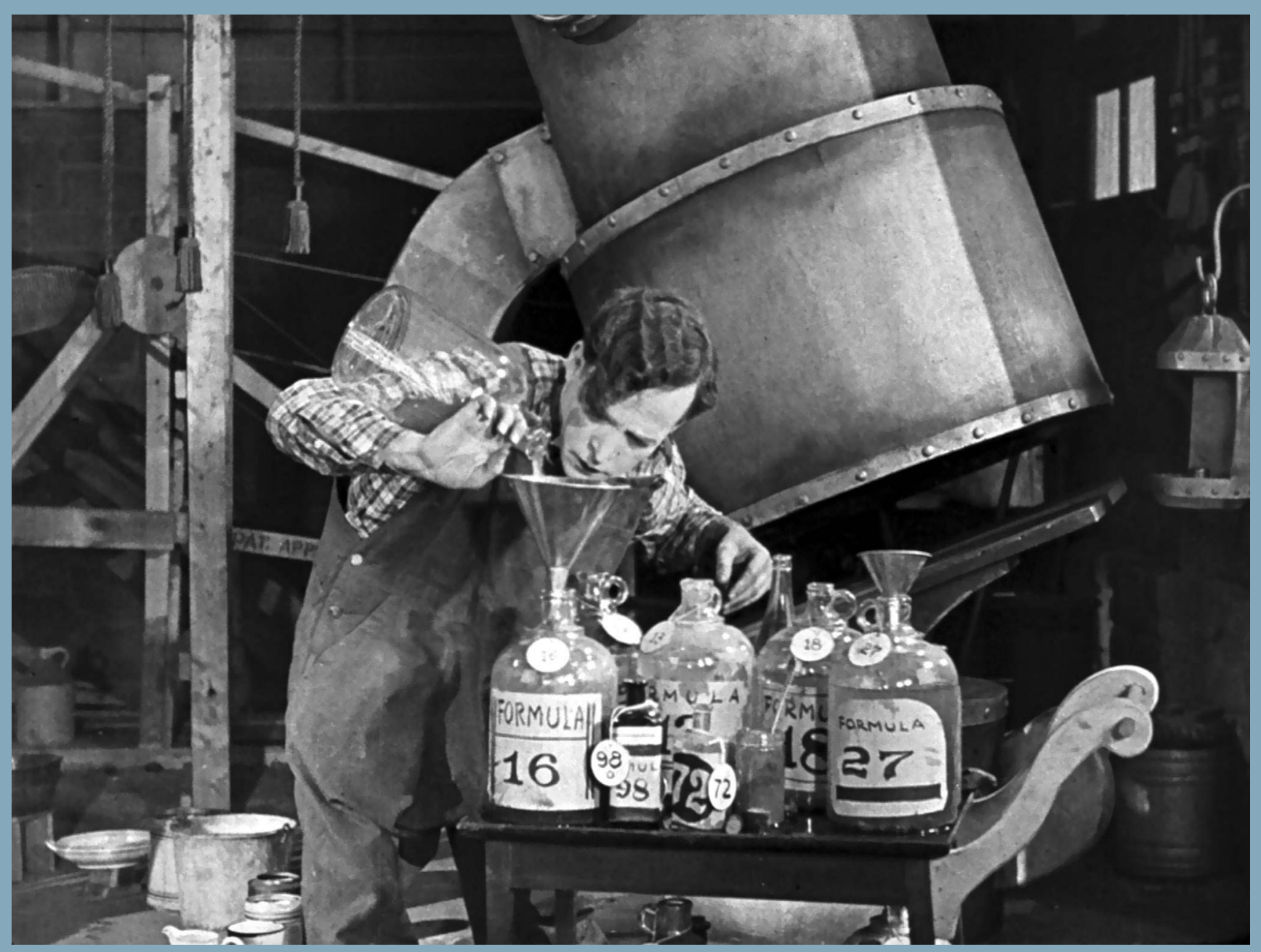




\section{Wild Tech}

\section{Repenser l'invention avec Charlie Bowers}

Lorsqu'il s'agit de rendre compte de l'histoire passionnelle que l'homme entretient avec les techniques, il est difficile de ne pas succomber à une forme d'évolutionnisme minimal. Du silex à l'ordinateur, l'homme ferait nécessairement travailler plus de neurones et entretiendrait un rapport plus complexe à la fois à l'objet lui-même, à la décision et à son environnement. Ceux qui, comme David Edgerton (The Shock of the Old, 2007) dénoncent le caractère illusoire de tels présupposés, faisant l'apologie du «low tech » contre le « high tech », démontrent que l'histoire des techniques ne peut se résumer à une courbe de complexification croissante, car les choix technologiques constituent toujours des exploitations partielles de capacités et certaines sont délaissées, d'autres favorisées. Mais ils n'évitent pas à leur tour le risque de la linéarité : l'histoire des techniques oscille toujours entre évolution et régression, le « low » et le « high », tentant de situer ses objets dans une chronologie ou au mieux sur un arbre phylogénétique. Toute tentative d'histoire globale des inventions finit toujours par se prendre à son propre piège. Et on se demande s'il n'est pas aussi incongru de vouloir comparer un silex et un robot qu'un dauphin et une libellule. De la même façon que l'un vit dans l'eau et l'autre dans les airs, les techniques ont souvent été poussées dans des directions profondément disparates. Toujours plus d'altération, d'estrangement, qui nous pousse à explorer d'autres sens, à contorsionner le corps, à prendre d'autres postures. Propositions expérimentales au service de projets de transformation hétérogènes, les inventions techniques sont souvent «wild » avant d'être « low » ou « high ». Dans ce domaine, une anthropologie qui chercherait à tenir ensemble le plus ancien et le plus moderne, le plus proche comme le plus exotique, le plus banal comme le plus étrange, ne peut se contenter de débusquer et de décrire des variantes. Elle doit pouvoir se prononcer sur la nature profondément expérimentale des rapports ainsi établis, en prenant en compte 
au lieu de les écarter d'emblée les «technicisations » qui troublent ou laissent perplexe, les usages aventureux, les circuits qui compliquent les chaînes opératoires au lieu de les simplifier, guettant ce moment où les techniques dérivent vers des « formes limites » d'elles-mêmes, se créolisent au point de se rendre méconnaissable ou s'épanouissent dans des directions que personne n'aurait pu prévoir.

Charlie Bowers (1889-1946), plus connu en France sous le nom de « M. Bricolo », est une figure originale et injustement méconnue du cinéma d'animation du début du xxe siècle. Je me servirai de Bowers pour mieux définir les contours de cette catégorie transgressive qu'on pourrait appeler le « wild tech», par rapport au « low» et au « high». Le propos de cet article n'est en aucun cas une biographie de Bowers, mais plutôt une tentative de reconstruction, à partir de ses films, de sa philosophie de l'invention dont on verra qu'elle est d'une étonnante modernité. De la vie de Bowers, on ne connaît pas grand-chose, à part qu'il fut un pionnier du cinéma d'animation et conçut un procédé optique d'animation appelé « Bowers Process » qui consiste à introduire image par image du dessin dans une situation réelle pour créer des effets magiques de métamorphose. Il est aussi l'un des premiers à expérimenter l'animation de créatures aussi imaginaires que bizarres, fabriquées avec les moyens du bord, inventant une animatronique de garage bien à lui. Les historiens du cinéma n'ont pas manqué de souligner les apports de cette figure oubliée au $7 e$ art, même si on connaît peu de chose de sa carrière et de la manière dont il fabriquait ses films. Son cinéma était peut-être trop burlesque pour être pris au sérieux. Bowers se met toujours en scène comme un inventeur, le voisin sympathique qui passe ses week-ends à bricoler au fond de son garage. Mais ce drôle d'inventeur est voué à un destin tragique, soit parce qu'il a des idées délirantes, soit parce que ses inventions ne marchent pas ou finissent par tourner à la catastrophe, une fois adoptées par des utilisateurs. Comment percevoir dans les aventures aussi risibles que dramatiques de M. Bricolo autre chose qu'un reflet des symptômes de notre civilisation ? Près d'un siècle plus tard, personne n'a jamais réussi à inverser ou à rediriger la course frénétique à l'invention que Bowers s'est plu à mettre en scène. Et pour cause, les techniques doivent se renouveler perpétuellement, être fabriquées et refabriquées. Le cinéma de Bowers illustre donc une tension cruciale de l'histoire moderne des techniques: les usines n'ayant pas le monopole des machines et les industriels de l'invention, n'importe qui peut concevoir avec un minimum d'outils et un peu de génie, mais pour concevoir quel monde ou quel environnement? Cette préoccupation qui était déjà bien prégnante aux États-Unis à l'époque de Bowers adopte des formes diverses au xxe siècle et se retrouve dans des mouvements plus récents de démocratisation des moyens du faire comme celui des makers et du Do it Yourself (Lallement 2015). Il est probable que si ces derniers connaissaient Bowers, ils feraient de « M. Bricolo», le personnage qu'il incarne, l'une de leurs figures cultes! Là n'est pas la seule raison qui doit nous inciter à prendre son cinéma au sérieux. Bowers est finalement l'un des grands théoriciens de l'expérimentation sauvage, du wild tech, esquissant une sortie hors de l'opposition stérile du high tech et du low tech. Ses inventions sont des « assemblages limites»: fragiles, elles flirtent avec les limites de ce que la technologie peut faire. Possédant par ailleurs une touche de réflexivité en plus par rapport à des techniques destinées au seul usage, elles 
interrogent le bien-fondé de l'invention et sont porteuses de promesses, de métamorphoses, qui mettent leurs usagers en situation expérimentale. Bowers arrive toujours à trouver des patrons, à convaincre des usagers potentiels pour ses inventions. Il ne reste plus ensuite qu'à faire le grand saut dans l'inconnu...

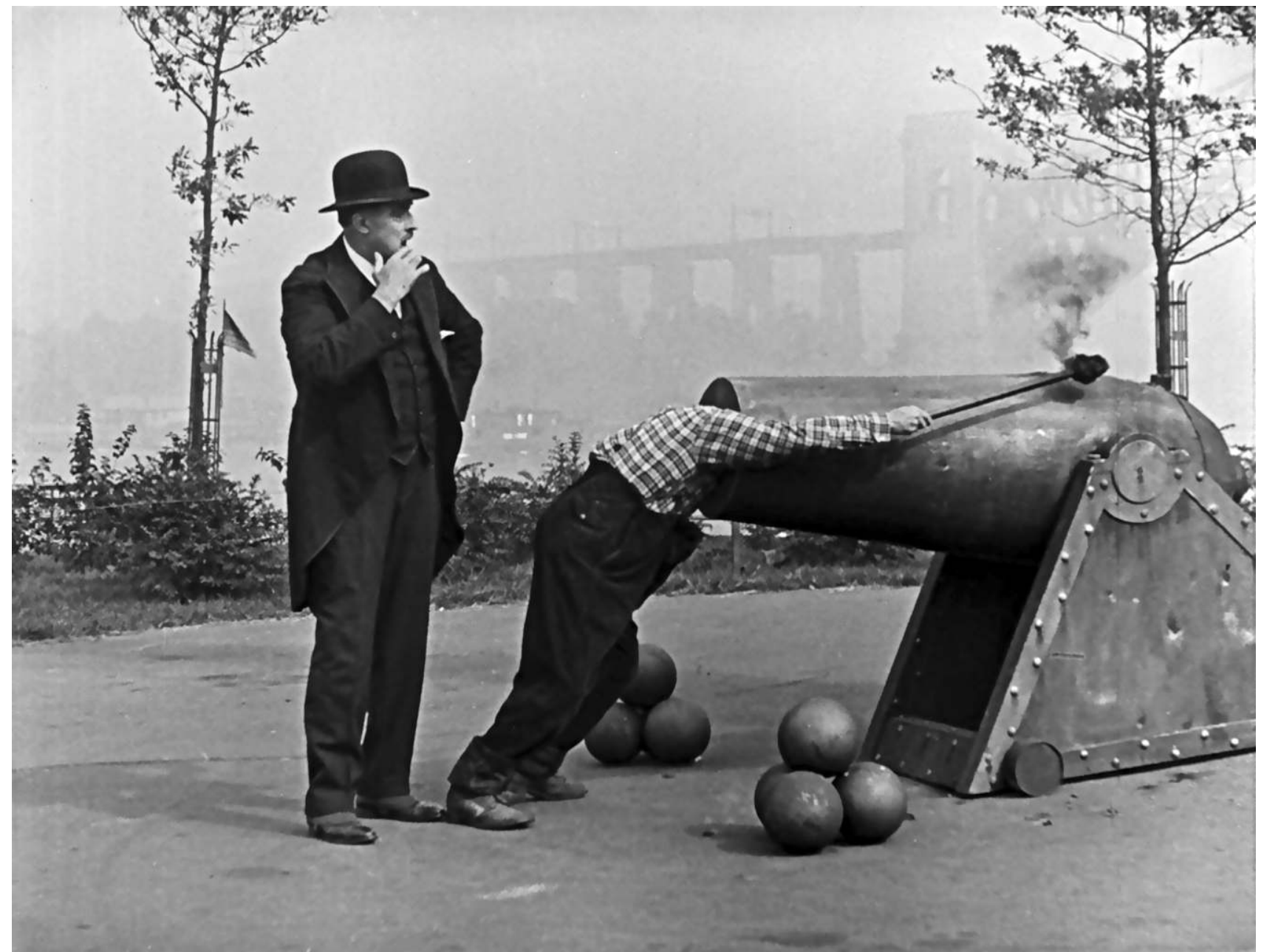

L'invention de machines excentriques censées résoudre des problèmes incongrus jusqu'à 1. - M. Bricolo. Non, tu leur implantation dans l'espace domestique (la maison, la rue) constitue l'un des ressorts fondamentaux du cinéma d'animation de Bowers. Ses bricolages ouvrent en tous sens et dans toutes les directions les entrées (input) et les sorties (output) des machines. C'est un ensemble de désirs, 
de demandes, de besoins qui ne sont pas listés par l'histoire conventionnelle des techniques qui se trouvent alors traités. Les machines destinées à la vente sont toujours exposées dans des espaces de démonstration très contrôlés, au mieux dans des espaces interactifs invitant l'utilisateur à en tester la viabilité dans des chaînes opératoires qui ne doivent pas les mettre en danger. Les films de Bowers ne se soucient pas de ces précautions. Ils simulent des inventions dont on peut suivre toute l'aventure, de leur conception à leur implantation dans un environnement, jusqu'à leur rejet ou leur destruction lorsqu'elles sont mal adaptées. Si les ingénieurs sérieux peuvent ignorer les " chaînes opératoires ${ }^{1}$ de Bowers sous prétexte qu'elles ne sont pas sérieuses, elles possèdent néanmoins une grande vertu : elles invitent à se poser des questions très saines à la fois sur la raison d'être des objets techniques et sur leurs implications.

\section{Cinétiques de la " circuitousness "}

Pour comprendre toute l'originalité de M. Bricolo, cet inventeur compulsif que Bowers incarne dans chacun de ses films, il faut partir d'une définition simple de la technique. L'anthropologue Alfred Gell nous en donne une qui a l'avantage de s'appliquer parfaitement à notre cas :

«Ce qui distingue la "technique" de la non-technique est un certain degré de circuitage [circuitousness] dans l'obtention d'un résultat donné. [...] Les moyens techniques sont des moyens détournés [roundabout means] d'atteindre un but. Le degré de technicité est proportionnel au nombre et à la complexité des étapes qui lient le donné initial à l'objectif poursuivi »² (Gell 1988; Stoichita, Grimaud \& Jones 2011).

Avec Bowers, le circuitage de l'objet technique se décuple au point de devenir absurde. Bowers partage ce goût de la provocation avec d'autres, notamment avec Rube Goldberg (18831970), dessinateur à la même époque de circuits et de machines se compliquant délibérément la tâche (Goldberg 2000 ; Spooner, Hunkin et al. 2000)³. Mais chez Goldberg et dans les concours de machines qui portent aujourd'hui le même nom ou encore dans ce qui est devenu au Japon une discipline à part entière, le chindogu (l'art de fabriquer des choses « non inutiles ») (Kawakami 1995, 1997, 2005), l'acte de circuitage vise toujours à compliquer ce qui peut être simple, multiplier et fragmenter les étapes, cultiver jusqu'à l'extrême la fragmentation et les circonvolutions pour elles-mêmes. Or chez Bowers, il ne s'agit pas tout à fait de cela. Certes, Bowers appartient à un genre de cinéma que l'on peut qualifier de burlesque, au même titre que Buster Keaton ou Snob Hubbard, mais les machines qu'il invente ne sont pas conçues pour le plaisir ou pour se compliquer la vie. Elles prétendent résoudre des problèmes que se posent les gens. Ce n'est pas par la manipulation (virtuosité du prestidigitateur qui répète ses tours de main) ou en ajoutant des pièces ou des rouages, en compliquant là où ce n'est pas utile (comme chez Goldberg), qu'il atteint son but. Comme on le verra, Bowers n'est ni réductible 
à un magicien ni à un bricoleur ou à un machiniste. Il est un peu de tout cela à la fois, mais il est aussi bien plus.

Un prestidigitateur impressionne les gens avec des tours dont on sait pertinemment qu'ils s'expliquent même si on ne sait pas comment. Bowers ne s'explique pas toujours le tour surprenant que prennent ses machines. Un machiniste ne fait qu'opérer, il ne conçoit pas. Bowers conçoit, opère et défend ses machines auprès de commanditaires divers. Mais Bowers n'est pas non plus un ingénieur comme les autres. Un ingénieur innove à partir d'un état de l'art de la technique de son temps. Ce que Bowers se propose de mettre en machine, en procédé ou en « circuit » est bien plus complexe. Il pratique un métier qui n'existe pas. C'est un « machinateur » de désirs. C'est en ouvrant les machines à des demandes multiples, parfois sensées parfois insensées, et en mêlant ensuite l'organique et le machinique dans des mécanismes qui court-circuitent les genres (animal, végétal, technique) qu'il opère. Se présentant toujours comme un solutionneur de problèmes, Bowers invite à machiner ce qui ne peut être machiné ou ne l'a jamais été.

On connaît peu de chose de la carrière de Bowers si ce n'est qu'il a eu un moment de gloire dans les années vingt, que Joseph Losey lui a rendu hommage comme animateur et qu'André Breton a vu dans un de ses films (It's a Bird), projeté dans un cinéma parisien en 1937, un chefd'œuvre. Bowers est mort injustement oublié. Cette injustice est d'autant plus troublante que Bowers a poussé à un degré de finesse rare des procédés qui ont ensuite été largement exploités par le cinéma d'animation du xxe siècle (mélange de prises de vues réelles et animées, stop motion, usage de marionnettes et de figures en pâte à modeler, etc.). C'est seulement récemment que Raymond Borde, directeur de la cinémathèque de Toulouse et amateur de vieilles pellicules, a retrouvé plusieurs de ses films dans des stocks ayant appartenu à des projectionnistes itinérants ${ }^{4}$. Des analyses du cinéma de Bowers, il y en a peu à l'exception de celle de William Solomon, professeur d'anglais de l'Université de Buffalo (New York), auteur d'un bel article sur Bowers. Solomon tente de le resituer ainsi dans la grande aventure moderniste :

« Bowers' use of the medium to exhibit a set of absurd machines that operated in unruly and unreliable ways enabled him to cut a deviant path through industrial modernity. Inevitably proving financially disastrous, his humorously dysfunctional creations generate laughter at the expense of the ethos of productive rationalism, in the process opening up an alternative understanding of machinery as the locus of exuberantly unsettling bursts of joyful play » (Solomon, 2010).

Exubérant, alternatif, drôle, inclassable même plusieurs décennies plus tard, tels sont les qualificatifs qui reviennent le plus souvent pour qualifier le cinéma de Bowers.

Mais sur quels présupposés reposent ses machines? C'est toujours le constat d'un manque ou d'un déficit qui pousse M. Bricolo à concevoir une machine. Tout le monde sait que les œufs sont fragiles et qu'ils se cassent. M. Bricolo se lance alors dans la conception d'une machine à rendre les œufs incassables. Le propriétaire d'un restaurant est abandonné par sa main-d'œuvre, M. Bricolo se met alors à bricoler une machine infernale qui permet d'accomplir toutes les tâches d'un restaurant, de la cuisine au service. Il manque une méthode qui permet de greffer tout à 
tout ou presque. M. Bricolo invente alors un procédé de greffe universelle qui permet de faire éclore des chats à partir d'une plante. La formule, d'un film à l'autre, ne change guère. À chaque fois en effet, c'est par amour que M. Bricolo invente ses machines, parce qu'il est amoureux et qu'il veut plaire à la fille du propriétaire de son restaurant ou à la fille d'un paysan dont le champ a été envahi par les souris. Mais chaque situation étant unique, la forme et les caractéristiques de la machine conçue pour résoudre le problème sont à chaque fois singulières.

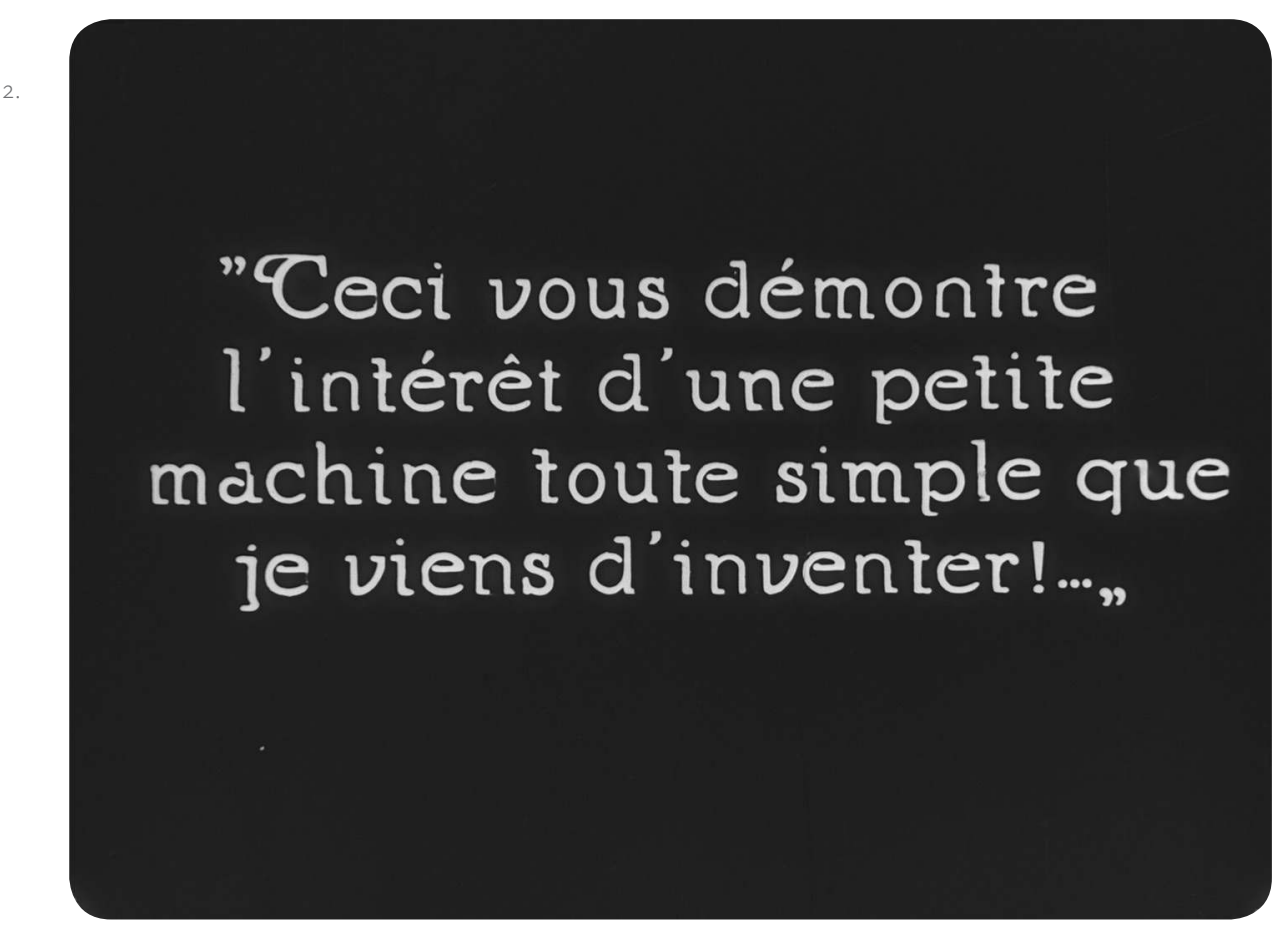

Chaque film de Bowers est centré sur une invention dont on suit le destin. Le film en examine la raison d'être, ses applications parfois dramatiques et la suit bien souvent jusqu'à ce qu'elle dérape. Dans Now, you tell one, où M. Bricolo clame avoir inventé son fameux procédé de greffe « universelle», on le voit arroser un arbre portant des fruits reliés entre eux par des tuyaux. Cet arbre incroyable enchevêtrant branches et tuyaux, donne naissance à des fruits « composés » par hybridation : un ananas sur lequel ont été greffés un oignon et un citron, par exemple. M. Bricolo découpe et replace des branches qui correspondent à des fruits différents, en les collant avec une substance qui ressemble à de la colle, qu'il sort d'un réservoir qui ressemble à un pot de peinture. Il colle la branche et met du scotch pour qu'elle tienne. Très vite sort de la branche une aubergine (en stop motion animation). M. Bricolo découpe l'aubergine et 
en laisse voir l'intérieur dans lequel se trouvent un œuf et une salière. Il teste la dureté de l'œuf avec une hache et mange l'œuf dur après l'avoir salé.

M. Bricolo réinvente la magie sympathique, les principes de contiguité, de similarité et de contrariété, « par relation préalable de tout à partie, ou par contact accidentel » comme disaient Hubert et Mauss (1902), qui traversent les pratiques magiques, derrière l'incroyable profusion de leurs liens. Et c'est parce que tous ces matériaux ne peuvent en réalité se marier ensemble que M. Bricolo les réunit dans une même chaîne opératoire, essentiellement optique. Le stop motion cinématographique permet de les mettre en contact et de matérialiser des processus de cause à effet impossibles. La séquence est invraisemblable, une suite de gestes cocasses et surréalistes, qui défie les principes du bricolage, mais réfléchissons-y un peu. Pourquoi avonsnous ce sentiment? C'est simplement parce que, d'expérience, nous savons qu'un arbre ne produit pas des œufs durs. Le jour où un arbre produira un œuf dur, le film de Bowers perdra sa dimension magique. On sait que pour qu'une greffe fonctionne, il est préférable d'avoir acquis une connaissance des plantes, de leur vie organique, et laisser la plante faire son travail, intégrer la greffe de façon à ce qu'elle fasse corps avec elle. M. Bricolo décale le principe de la greffe en important des matériaux qui lui sont étrangers et accessibles à tous (colle et scotch) et en simplifiant la méthode de la bouture ; intégrant l'aléatoire comme une donnée fondamentale, la greffe donne lieu à des résultats imprévisibles, l'arbre dégorgeant d'objets que Bowers n'avait pas prévus. Des liens de causalité impossibles sont projetés aux yeux du spectateur tandis que $\mathrm{M}$. Bricolo se fraye un chemin improbable entre les règnes (végétal, animal, objectal). Les assemblages de Bowers sont toujours hybrides par leurs matériaux, ils ne sont jamais purement faits de métal ou de bois, ils mêlent des ordres a priori difficiles à fusionner et exploitent souvent un maximum d'incompatibilités afin de générer l'effet de surprise. Les animaux et les plantes font bon ménage ici avec l'acier et la colle. L'arbre de M. Bricolo compose avec une notion de greffe bien plus ouverte, souple et ignorante des véritables principes de la nature que celle des botanistes, des agronomes ou des biologistes. Tandis que ces derniers ne réalisent que des greffes partielles, ce qui intéresse M. Bricolo est d'inventer un principe « universel » de transplantation dont les applications seraient sans limite de forme ni d'objet. Pour cela, Bowers mélange les genres dès le début : la botanique avec la tuyauterie, la nature avec le collage, mais surtout il fait se rencontrer dans un même circuit, des éléments qui ne peuvent se trouver liés dans un processus horticole classique. La puissance du stop motion est de permettre une forme de simulation optique concrète : des enchaînements de cause à effet relégués d'ordinaire dans l'impossible sont un peu plus appréhendables quand on les voit ainsi prendre forme devant nos yeux. Le circuit optique final - « d'un arbre jaillit un œuf dur prêt à consommer par l'intermédiaire d'une aubergine » - ne fait pas seulement écho aux « chaînes sympathiques » de la magie décrites par les anthropologues. Elle évoque aussi les détournements surréalistes des machines de Munari, par exemple, qui exploitaient le même principe de mise en contiguité de corps a priori incompatibles. Celles-ci intégraient à l'intérieur d'une même chaîne opératoire les choses les plus hétérogènes, y compris des êtres vivants et en particulier des animaux, liées par actions et réactions ou stimuli et réponses. Mais Munari ne va pas aussi loin que Bowers, notamment 
parce qu'il ne fait que dessiner ses machines sur le papier, alors que Bowers les fabrique, certes pour le cinéma, mais il doit tout de même les rendre opérationnelles, optiquement viables, à l'intérieur de « chaînes sympathiques » d'images liées entre elles.

La suite du film est intéressante, car Bowers va faire la tournée des fermes pour vendre son procédé de greffe universelle. Il se met en scène en train de tester sa machine dans des situations diverses pour en éprouver la viabilité (ou l'impraticabilité). Et c'est là que son cinéma s'avère être bien plus qu'une réappropriation cinématographique de la « sympathie par contiguïté » au sens d'Hubert et Mauss. En magie, nous disent ces auteurs, « on limite toujours les effets de la sympathie à un effet voulu. D’autre part, on interrompt, à un moment précis, le courant sympathique ; d'autre part, on ne transmet qu'une, ou un petit nombre des qualités transmissibles » (p. 41). Comme souvent chez Bowers, le test ne fonctionne pas, parce que son invention le dépasse, sa greffe marche toute seule et n'en fait qu'à sa tête, elle devient incontrôlable, greffant tout à son insu, sans faire de distinctions. Le procédé une fois mis au point suit son mouvement propre, indépendamment de son inventeur. Il déchaîne une force que personne ne pouvait soupçonner et se déploie souvent avec une violence incroyable. Un arbre de Noël pousse sur le manche d'une brouette d'un paysan qui se met alors en colère. Pour sauver une famille dont les plantations ont été envahies par les souris, M. Bricolo décide d'appliquer son processus de greffe universelle pour créer des chats. C'est alors plusieurs milliers de chats qui jaillissent d'une plante de manière incontrôlable. Et M. Bricolo finit chassé à coups de balai de la propriété de celui qu'il était venu sauver. À la fin du film, qu’applaudit-on ? La prouesse d'une invention impraticable ? La révolte des objets contre leurs inventeurs et usagers ? Les déboires d'une technologie qui rend un culte à la mécanicité des effets, alors qu'elle ne finit pas d'en déclencher d'autres impossibles à prévoir ? Sans doute un peu tout cela à la fois. Bowers nous prouve que son invention peut être entrevue, ressentie et appréciée à un certain niveau qui n'est pas celui d'un rapport ordinaire, purement fonctionnel au procédé technique. La greffe de $\mathrm{M}$. Bricolo ne peut pas fonctionner selon les critères de la science et de la botanique, mais elle fonctionne dans les termes de M. Bricolo, elle est viable dans le cadre d'une « animation optique ». Le processus d'éclosion de la greffe est une suite d'apparitions. Des objets que l'on n'imagine pas émerger les uns des autres le peuvent, une fois traités comme les « composantes optiques » d'une animation. Ils peuvent alors éclore les uns des autres, au sein d'un processus qui repousse toujours plus loin les limites ce qui peut être lié ${ }^{5}$. Toute l'originalité du « Bowers Process » est là. Et c'est sans doute ce qui fait que Bowers, plus que tout autre, célèbre le cinéma comme méthode de simulation, visant à simuler « ce qui se passerait si... », effleurant par l'œil des possibilités que l'on ne pourrait envisager autrement. Le «Bowers Process », ce procédé optique de mélange de prises de vues réelles et d'animation, permet toutes les hybridations. En réalité, l'invention de Bowers et celles de $\mathrm{M}$. Bricolo se répondent, s'alimentent au point de n'en composer bien souvent qu'une seule. Ce dont $\mathrm{M}$. Bricolo va chercher à convaincre les paysans qui l'entourent, par exemple, c'est de la valeur de sa technique de greffe optique, bien plus ouverte et surprenante qu'une technique de greffe normale. Mais il faut les dissocier pour comprendre comment Bowers fonctionne. Lanimation, c'est la réponse de Bowers à l'imagination 
délirante de M. Bricolo et c'est la seule réponse possible dans l'état actuel des techniques. D’une plante, personne n'a encore réussi à faire éclore des chats. D'un œuf, on n’a jamais vu émerger des voitures, sauf dans les films de Bowers.

La machine à automatiser les tâches d'un restaurant, conçue par M. Bricolo dans He's done his best, propose une autre chaîne opératoire toute aussi intrigante que les précédentes. Lanimation optique est assumée encore plus ici que dans le cas précédent comme une forme ultime de magie sympathique qui secoue la technique sur ses fondements. Bien plus qu'une simple machine, il s'agit d'un véritable terminal changeant à jamais le statut du cuisinier et des matériaux pouvant entrer dans un processus de composition culinaire, une centrale de contrôle anticipant le triomphe du « génétiquement modifié », capable de transformer tout ce qui existe en nourriture potentielle, afin de répondre aux commandes des clients. Une illustration, au trait à peine forcé, de la pulsion « transgénique » qui hante la filière agroalimentaire. Une main aux gants blancs fait éclore une boîte de petits pois à partir d'une plante, des tas d'objets (y compris une chaussure) passent dans un hachoir qui en fait de la viande hachée, une huître comprend par elle-même qu'elle doit parfumer une soupe. Sans cette main à tout faire, plus habile et intelligente qu'une main humaine, sa machine ne fonctionnerait pas. C'est elle qui gère la diversité des opérations de conception en cuisine. Cette machine n'est pas seulement révolutionnaire parce qu'elle oblige à repenser le partage ordinaire des tâches de la restauration, centralisées autour d'une main magique. L'excentricité de cette machine absolue tient à sa double ouverture, en amont et en aval du geste de conception : elle est infiniment ouverte sur l'hétérogénéité des ingrédients potentiels et peut répondre à toutes les demandes.

On peut rejeter Bowers, sous prétexte que l'aubergine qui pousse d'une branche, l'œuf qui jaillit d'une aubergine, la colle qui sert à coller les branches, l'arbre traversé par des tuyaux en plastique ne peuvent jaillir que du cerveau d'un poète. Mais il ne faut pas croire que les ingénieurs ne partent pas, eux aussi, de demandes incongrues pour élaborer leurs machines. Toute une philosophie de la conception (Lallement 2015) ${ }^{6}$, à l'œuvre dans les « fablabs », les écoles de design et d'ingénieurs vivement encouragés à donner libre cours à leur imagination pour inventer les objets de demain, mêle les préoccupations pragmatiques à des techniques « bowersiennes » d'invention, jouant avec des principes contre intuitifs et des matériaux a priori incompatibles. Le succès des concours de mécanismes «à la Rube Goldberg », ces mécanismes qui ne se donnent aucune limite de matériaux ni de connexions possibles, dans les écoles d'ingénieurs américaines, en est la preuve. Rejeter Bowers sous prétexte qu'il ne se donne au fond aucune limite dans ses inventions, c'est mal comprendre la vertu essentielle des machines burlesques et plus généralement du wild tech par rapport au low et au high : elles permettent d'entrevoir et d'évaluer très concrètement, à une époque donnée, quelles sont les limites du possible et d'identifier les zones qui résistent à toute tentative de technicisation. Bowers propose une sorte d'alliance entre la magie et le design, un pacte entre le « mécanique » et le «sympathique » qui est loin d'avoir perdu sa pertinence tant leur lien est constamment à repenser, dépendant de l'état historique des possibilités et des impossibilités techniques. Il nous projette par ailleurs dans un monde où les moyens et les désirs de fabriquer du procédé sont bien plus distribués 


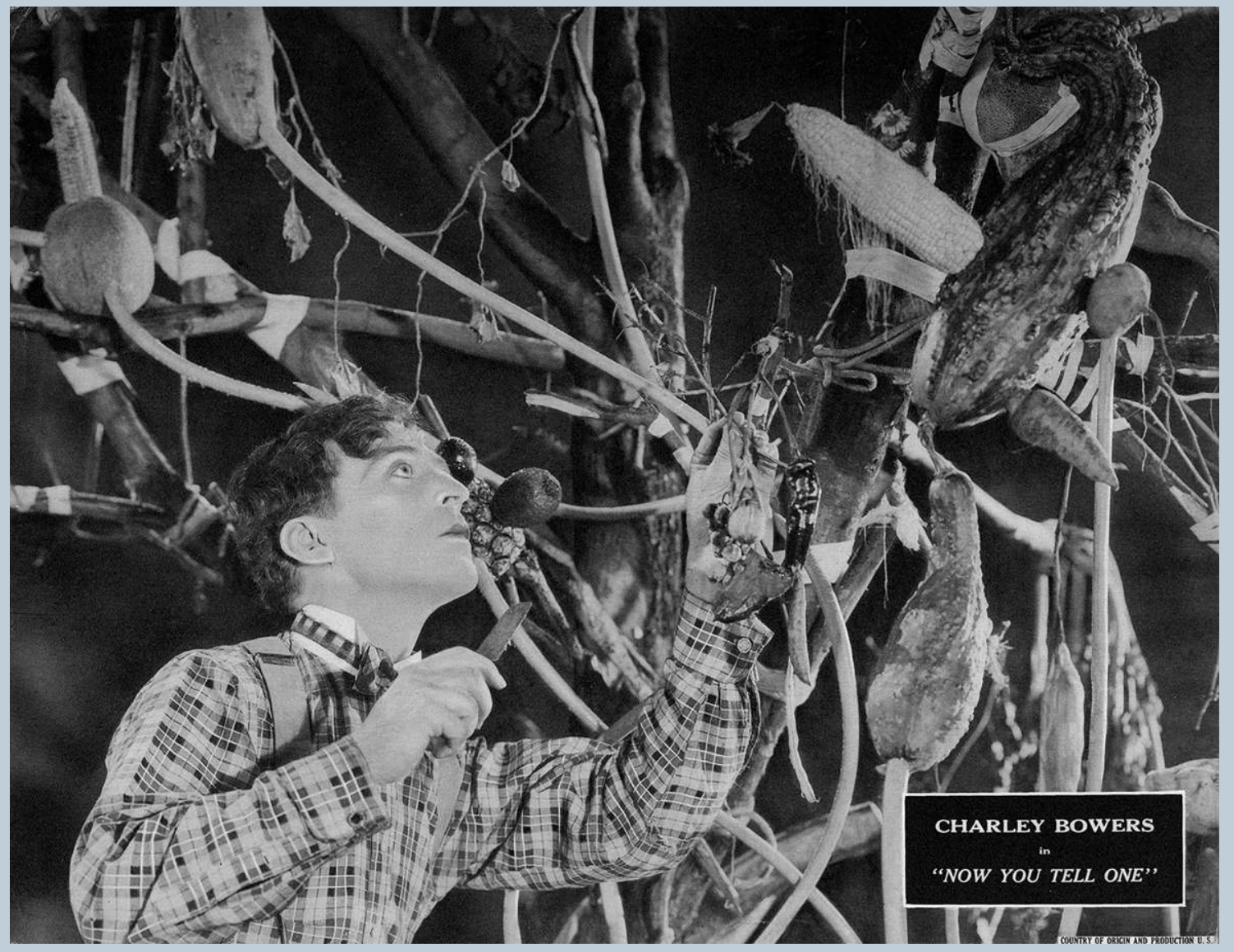




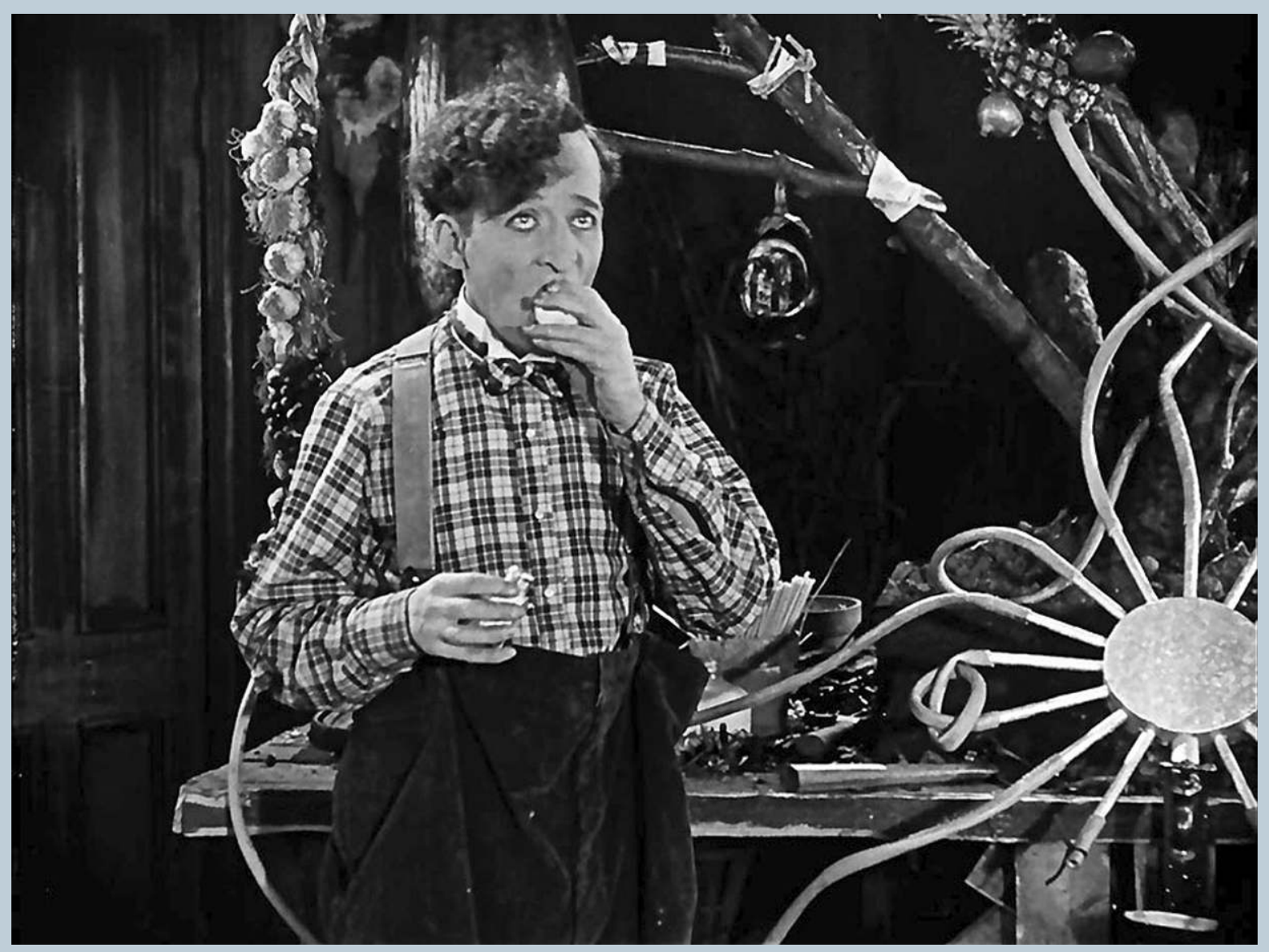


et ne sont pas seulement entre les mains des ingénieurs reconnus comme tels. Comment les adeptes du Do It Yourself ont-ils pu ignorer que ce problème ne date pas d'hier et avec lui, la question des limites mêmes de l'invention? Avec Bowers, ce qui entre dans la composition d'un procédé n'a pas de limites. M. Bricolo, ne répond pas à une demande prototypique mais à une multitude de désirs singuliers, fluctuants et qui aboutissent à autant de procédés et de circuits qu'il y a de possibilités de machiner ces besoins.

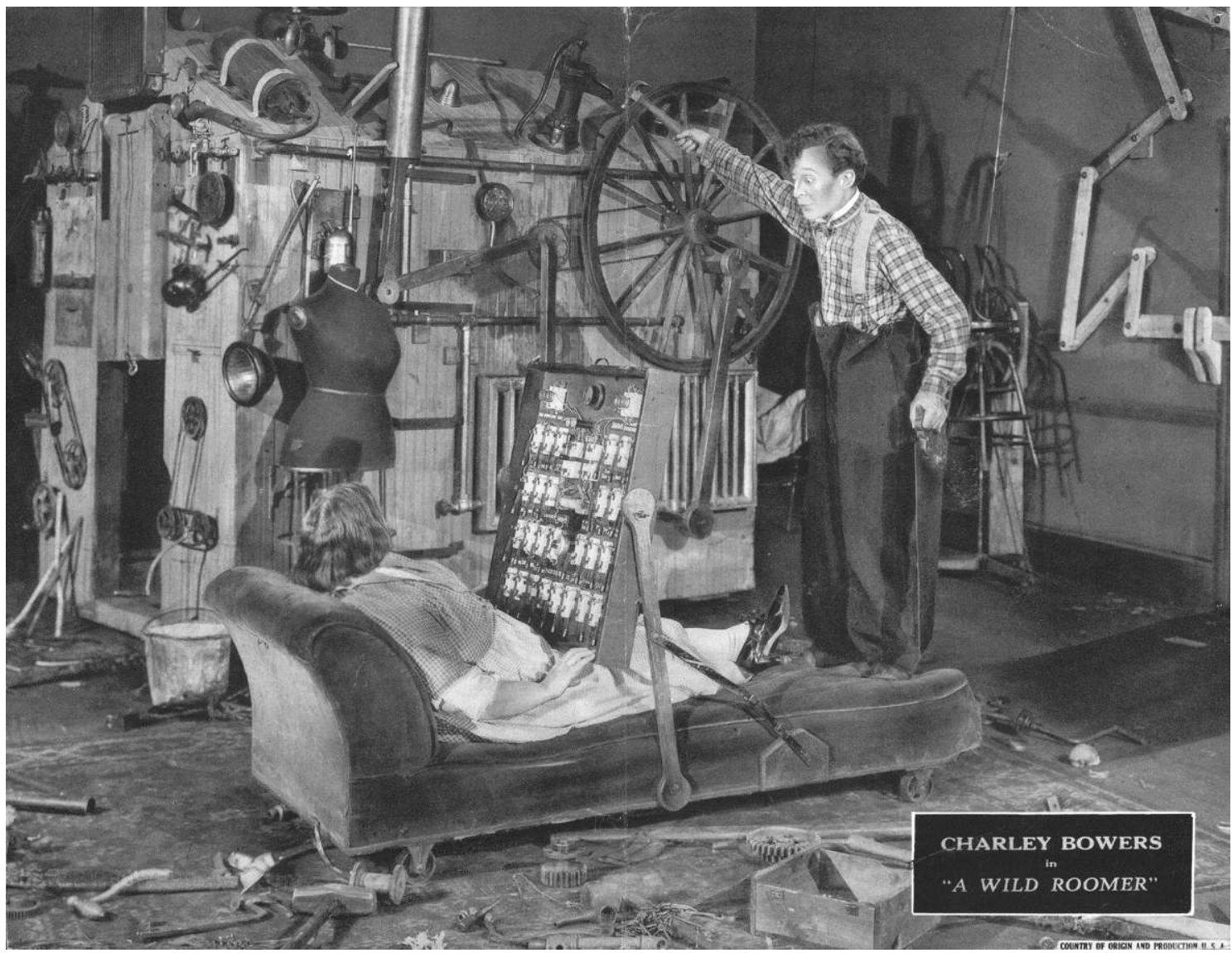

\section{Machinisme ambulant et inventions de proximité}

On comprend dans ce contexte que les films de Bowers ne se limitent pas à une critique de la société industrielle des années vingt ou à l'anticipation des problèmes posés par la démocratisation des «moyens du faire ». L'acte même d'inventer est au cœur du projet critique de Bowers. 
M. Bricolo finit toujours par être la victime de ses propres inventions et on prend goût à voir son procédé de greffe déraper dans l'absurde, éprouvant de l'empathie à son égard lorsqu'il est lui-même ébahi par ses circuits constamment dérapant. Solomon qualifie son cinéma de "slapstick modernism [...] in which a series of willfully undisciplined acts of cultural improvisation defied the priorities governing large-scale capitalist manufacture $»$ (p. 171). Mais on peut aller plus loin. Bowers n'a rien à voir avec Chaplin. Inventeur marginal et nomade, il ne serait rien sans son garage ambulant ou sans son laboratoire qu'il promène d'un film à l'autre.

Si les dérapages de Bowers sont toujours singuliers, voyons comment il s'y prend pour se donner le maximum de chances de les limiter. Bowers intervient dans des maisons diverses, dans les pensions où il est locataire. Il peut à chaque fois se composer un garage, un laboratoire ou un atelier au sous-sol. Il pratique « l'invention de proximité ». Il cherche à résoudre des problèmes et il est toujours un peu en avance sur la demande. Quand il invente sa machine à automatiser toutes les tâches d'un restaurant (dans He's done his best) ou celles d'une maison (dans A wild roomer), personne ne lui demande rien, il anticipe les usages en même temps qu'il les crée. Dans tous les contextes où il intervient, il identifie des failles ou des creux dans les usages, des «zones muettes» qu'il se propose de peupler avec un procédé ou une machine. Le cinéma de Bowers a tout d'une mise en scène de la technique comme activité de résolution de problème. C'est dans des espaces ordinaires - l'espace de la maison, le champ du paysan, le restaurant d'à côté -, que $\mathrm{M}$. Bricolo est amené à exercer ses capacités d'invention, à débloquer les usages ou en anticipe de nouveaux, en y introduisant du procédé, de la machinerie. On pourrait penser que tous les problèmes n'ont pas une solution machinique, mais M. Bricolo est convaincu du contraire. Tout problème, aussi banal soit-il, constitue le prétexte à injecter de la circonvolution technique dans la vie des gens.

De quoi la boîte à outils de M. Bricolo se compose-t-elle? Son métier, c'est «inventeur », et à ce titre, son outillage est tout ce qu'il y a de plus hybride, du marteau à l'éprouvette de chimiste en passant par le microscope. Machiniste ambulant, Bowers ne cesse d'osciller entre le dépanneur, le mécanicien et le chimiste, pris dans une routine d'expérimentation tout en étant toujours à deux doigts de faire une découverte majeure. Et c'est quand il doit faire la démonstration de ses procédés plus ou moins rocambolesques qu'il se met en situation d'échec. Par conséquent, c'est moins la culture industrielle que Bowers prend pour terrain de jeu que celle de l'invention scientifique et technique en général, avec ses expérimentations en laboratoire et ses démonstrations devant des publics de testeurs. Alors que les laboratoires ont confisqué la notion même d'« invention », Bowers se la réapproprie. La longue chaîne de l'innovation scientifique se trouve repensée dans le garage laboratoire ambulant de Bowers, grand précurseur du Do It Yourself et de la « biologie de garage » (Meyer 2012). Expérimentateur tous azimuts, il met au point des formules qui ressemblent à celles des vrais laboratoires et qui se traduisent ensuite par des applications concrètes quasi instantanément. M. Bricolo ne reste jamais très longtemps dans son laboratoire, il a besoin d'expérimenter ses procédés très vite avec des cobayes, dans des situations réelles, même lorsque son procédé n'est pas encore au point. 
Lorsqu'il invente la banane antidérapante dans Many a Slip, c'est après avoir identifié par un «bricoloscope » (une sorte de microscope plus puissant), le « microbe du glissement » et tenté d'appliquer aux peaux de bananes le système du frein moteur. La machine à faire tremper les peaux de bananes dans les diverses solutions qu'il a composées est une sorte de grand manège de tiges permettant de tester en même temps un grand nombre de peaux. On ne peut s'empêcher de penser qu'il y a de l'inventivité dans cet appareil à faire tremper de multiples objets en même temps dans des solutions distinctes. Pour tester sa solution, M. Bricolo a besoin d'un simulacre d'escalier, mais aussi d'inventer un système qui permet de déposer régulièrement des peaux de bananes sur l'escalier de la maison, depuis son laboratoire qui se situe au sous-sol pour les tester in the wild. Ainsi Bowers invente le "machinisme ambulant», une forme de service qui n'est pas sans poser quelque problème logistique. Malheureusement comme à chaque fois, l'histoire finit par une grosse déception. Un homme d'affaires a appris la nouvelle de l'invention et s'apprête à faire un chèque à son inventeur. L'homme est en réalité un fou échappé de l'asile, très vite rattrapé par son gardien. Ultime pirouette, la propriétaire exténuée par les excentricités de M. Bricolo demande au gardien s'il reste de la place à l'asile dans la section des fous furieux. Et au lieu d'embarquer M. Bricolo, c'est la femme qu'il embarque...

Toutefois, on passerait à côté de l'originalité de Bowers si on réduisait son cinéma à une critique de la culture du laboratoire. Les machines de Bowers entraînent leurs usagers et le spectateur dans des usages bien plus réflexifs que des machines ordinaires dans la mesure où elles logent de la technique là où on pourrait aisément s'en passer. C'est peut-être une des singularités du wild tech par rapport au low tech. Tandis que le second s'appuie sur l'idée qu'on peut faire plus simple et plus économique pour répondre à des besoins, le premier introduit du procédé dans d'innombrables domaines de la vie à des fins purement expérimentales. Et pour cela, M. Bricolo a besoin de défragmenter les situations dans lesquelles il va introduire son procédé pour les réassembler autrement. La technique n'est rien d'autre ici qu'une opération de « contorsion » poussée à l'extrême. Le machinisme ambulant de M. Bricolo dérange les milieux où il s'exerce, il introduit des perturbations radicales, conduisant la machine sur un terrain où l'histoire des techniques l'a rarement conduite.

Si tout problème a une solution machinique pour M. Bricolo, il faut s'attarder sur A wild roomer, l'un des rares films de Bowers où la machine qu'il invente fonctionne sans se traduire par le rejet de l'inventeur hors du circuit qu'il a patiemment contribué à établir et même si elle marche encore une fois à l'insu de son inventeur. Il habite ici une pension dans laquelle les locataires subissent des phénomènes étranges. Des objets disparaissent, un meuble de cuisine au moment où la cuisinière pose sa théière dessus, la baignoire au moment où un locataire s'apprête à prendre son bain, l'appareil télégraphique au moment où l'utilisateur tape son message, l'appareil de musculation d'un locataire au moment même où il fait ses exercices. Les locataires se réunissent alors et pensent que M. Bricolo, surnommé ici «l'abruti du cinquième » (Knucklehead Charley), inventeur au génie méconnu, en est responsable. C'est que M. Bricolo est en train de concevoir une machine étonnante qui contiendra en elle toutes les fonctions possibles et rendra obsolète la fragmentation des appareils, des haltères à la baignoire en passant par le rasoir ou la 
machine à laver. Tout sera réuni dans une même centrale. M. Bricolo reçoit alors un télégraphe qui le rappelle d'urgence auprès de sa famille. Son grand-père décédé lui délègue toute sa fortune à condition qu'il fasse la démonstration de sa machine dont il lui a montré les plans avant de mourir. Dans le cas contraire, il perdra l'héritage au profit de son oncle qui s'en frotte déjà les mains. M. Bricolo retourne alors dans sa pension et force sa propriétaire à tester sa nouvelle machine révolutionnaire. Il s'agit d'une machine à automatiser toutes les tâches de sa pension, du lavage du linge au cirage des chaussures en passant par la manucure, le rasage, le balai, la cuisine, la gymnastique. La machine ressemble à un gros bunker muni de tuyaux, de robinets et de poulies connectés les uns aux autres et qui tournent dans plusieurs sens. M. Bricolo force sa propriétaire à s'allonger sur un divan et à prendre les commandes d'un tableau de bord (le tableau de la Ménagère à tout faire). Il ne s'agit pas seulement de tâches que la propriétaire se doit d'effectuer mais bien de toutes les tâches que tous les habitants de la pension réalisent quotidiennement. On voit surgir alors des mains d'une extrême délicatesse, munies de gants blancs animés par des tiges de métal, qui donnent naissance à une poupée jouant avec un écureuil.

On ne peut s'empêcher de penser que cette action d'une grande poésie et qui n'a rien à voir avec une opération mécanique n'était pas prévue et que cette machine pourrait bien être douée d'une forme d'intelligence magique. La plupart des circuits de Bowers impliquent à un moment ou à un autre de telles connexions, grâce à l'animation image par image (stop motion). Mais la machine de la ménagère idéale est aussi capable de torturer quiconque s'y frotte. Bientôt, elle est tellement lourde et compliquée que M. Bricolo est obligé de scier le plancher pour la faire descendre jusqu'au garage et il la conduit alors comme une voiture pour la déplacer jusqu'à la maison de son oncle où il est censé en faire la démonstration. M. Bricolo conduit sa machinerie ambulante à travers les rues, malgré les tentatives de son oncle de faire sauter le véhicule à la dynamite tout au long du chemin, dans ce qui est sans doute l'une des séquences les plus belles du cinéma de Bowers. Arrivé à la maison de sa belle-mère, il lui demande de se mettre au tableau de la ménagère et de choisir n'importe quelle tâche. L'oncle s'approche de la machine pour la détruire, mais celle-ci se transforme alors, sous l'impulsion de la belle-mère aux commandes, en véritable instrument de torture. Tandis qu'elle teste chaque bouton avec entrain, l'oncle se fait attraper et essorer violemment par la machine (bouton « essorage »), laver, secouer et raser brutalement (bouton « rasage »). Et après l'avoir promené dans la ville, la belle-mère jette l'oncle en toute inconscience dans la rivière, comme une vulgaire poubelle (bouton « déchets »). C'est donc par inadvertance, en ne sachant pas ce qu'elle faisait réellement et en appuyant simplement sur des boutons par curiosité, que la belle-mère de M. Bricolo s'est muée en bourreau. Ainsi, la centrale de l'intelligence domestique conçue par Bowers capable précédemment de faire éclore des poupées et des écureuils, rétrograde au final à l'état d'engin de torture mécanique, grâce à l'intervention d'un usager inconscient. Peupler les maisons comme le fait Bowers de machines et de procédés, revient à injecter un degré de complexité non nécessaire qui produit des effets inédits et imprévisibles. M. Bricolo ne peut ensuite qu'assister à la déroute de ses propres inventions qu'il ne contrôle pas. Il aspire à poétiser le milieu, mais ses projets se heurtent à un principe de réalité, celui de l'usage effectif. Et c'est l'usage, moins le sien que celui des autres, 
ultime acte de contournement, qui tranche. C'est pourquoi il faut voir dans Bowers bien plus qu'une critique de l'invention, un exercice de simulation, un véritable cinéma d'« anticipation des inventions » et de leurs implications. Il en donne à voir en temps réel, dans le cadre d'une animation minutée, les prémisses, les usages et les déroutes.

\section{Ces forces que la technique ignore}

Si chaque film de Bowers peut être vu comme la simulation d'une machine en exercice, on a bien vu comment M. Bricolo milite pour un certain type d'invention. Non pas le grand machinisme industriel, mais la machinerie artisanale, le bricolage, le collage éclectique. M. Bricolo est l'une des plus belles expressions cinématographiques des « laissés pour compte » de l'invention qui rêvent de déposer des brevets sans jamais y parvenir, bien loin des cercles reconnus et confinés de l'ingénierie et de la science. À l'époque de M. Bricolo, le « sans fil » (wireless) est un champ prometteur. L'Electrical Experimenter, revue créée par Hugo Gernsback en 1913, invitait les gens à se saisir de la radio, de l'électricité et militait en faveur de l'électricien amateur. M. Bricolo offre de l'amateur inventeur une version burlesque, mais ses interrogations sur la nature des forces qu'il manipule dans son « transgénisme de garage » sont d'une grande modernité. C'est qu'en réalité, il y a toujours dans ses procédés ou dans son entourage quelque chose qui vient décaler l'esprit de son invention ou bien carrément perturber la totalité de son plan d'origine et qui fait qu'il est impossible de nouer un pacte complètement rationnel avec la technique. Bowers joue beaucoup sur le fait que toute technique, quel qu'elle soit, met en œuvre une forme d'irrationalité du point de vue de son usager, des connexions sans fil, et qu'on ne peut jamais s'expliquer la totalité de son fonctionnement.

Interrogeons-nous sur ce motif récurrent de la pensée magique de Bowers : « d'un œuf émerge une voiture ». L'ouf et la voiture sont des ingrédients indispensables de la «formule Bowers », les matériaux de prédilection de ses animations image par image, réunis ici dans un unique processus de génération optique. Chez Bowers, les objets ne cessent de se métamorphoser en autre chose qu'eux-mêmes - de l'animal au végétal, du végétal ou de l'animal au mécanique -, mais l'opération n'est jamais mise en scène comme un miracle, elle est présentée au contraire comme le produit d'une pensée scientifique qui se serait laissé entraîner malgré elle vers un «transgénisme » généralisé. Les œufs qui se transforment en voitures sont un fait qu'il ne s'explique pas et dont il n'est pas responsable, un miracle qui se produit très souvent dans l'environnement de M. Bricolo, un procédé technique qui n'a pas d'auteur bien identifiable et qui est toujours d'une certaine manière « en avance » sur lui et vient perturber ses plans. Dans Eggs On, M. Bricolo invente une machine à rendre les œufs incassables. Il est convaincu du côté révolutionnaire de son invention et cherche à convaincre les distributeurs d'œufs d'acheter son invention. Sa machine, fabriquée au fond de la grange de son beau-père, est un mécanisme impressionnant à la Rube Goldberg mêlant des roues de vélo à des poulies, des tuyaux, des 
paniers et des marteaux. L'œuf entre dans la machine et évolue, telle une bille, dans un circuit complexe. M. Bricolo pédale avec enthousiasme pour en actionner tous les rouages. L'ouf est alors traité, testé et consolidé d'un mécanisme à l'autre, jusqu'à obtenir une peau en caoutchouc en guise de coque. Le tout marche à merveille jusqu'au moment de la démonstration officielle. M. Bricolo est en manque d'œufs. Il va les chercher dans les fermes environnantes et ne tombe au mieux que sur des poules qui pondent mais dont les œufs se transforment en voitures. Une armée de petites voitures vient même se réfugier sous une grosse voiture qui se met à les couver. M. Bricolo finit par trouver un œuf, mais il est l'œuvre d'une poule qui a pondu sur de la dynamite. Il passe l'œuf dans sa machine et au moment de tester sa dureté, l'œuf fait exploser sa machine. Bowers et ses clients potentiels peinent à se relever, noirs de suif, au milieu d'un paysage apocalyptique de pièces détachées. Quand on y regarde bien, ce n'est pas la machine qui a échoué dans ce cas précis. Elle a fonctionné. C'est la présence dans l'environnement d'un nouveau type de connexion, entre la nature et la civilisation mécanique, une greffe qui s'est faite toute seule loin de toute volonté humaine et qui se retourne contre l'inventeur de machines. Tandis qu'il cherche à machiner un produit animal, M. Bricolo se heurte à un animal déjà machiné au point de pondre des voitures puis des œufs remplis de dynamite.

Dans un autre film, Say Ah-h, M. Bricolo est l'employé d'une ferme, préposé à l'élevage des autruches. Il nourrit une autruche avec du ciment qui pond mais ses œufs sont impossibles à manger pour son propriétaire furieux. Un jour l'autruche pond, et l'autruche qui sort de l'œuf est munie d'un balai à poussière en guise de queue et mange tout ce qui est disponible dans le garage de M. Bricolo, y compris du métal. Elle ne fait aucune distinction : outils, ustensiles, déchets, matériaux, sacs de ciments, elle avale tout, même le non recyclable. Elle se révèle aussi capable d'apprécier la musique et de se choisir un disque au gramophone. Cette autruche mélomane, un peu civilisée mais pas trop et surtout très gloutonne, donne naissance ensuite à d'autres autruches mangeuses de métal. Il ne faut pas voir dans ces figures d'oiseaux recycleurs auxquelles Bowers a souvent recours une simple revanche de la nature sur la culture. C'est plutôt l'anticipation d'un monde d'organismes génétiquement modifiés mais sauvages et capables de se reproduire eux-mêmes hors contrôle. Bowers rejoint ici les préoccupations d'un Jacques Lafitte, auteur de Réflexions sur la science des machines (1932), qui décrivait des lignées de machines comme on peut décrire des espèces biologiques. Certes, on pourra toujours se plaindre que les manufactures d'automobiles ont remplacé les fermes d'antan, que rares sont les œufs qui n'ont pas été trafiqués et que les voitures se multiplient comme des poussins. En « hybridant » l'œuf et la voiture, Bowers amène son spectateur à s'interroger sur la filière agro-alimentaire autant que sur l'industrie automobile. Mais le plus surprenant, c'est que M. Bricolo qui connaît bien des choses, malgré l'excentricité de ses inventions, ne s'explique jamais cette hybridation et la subit comme une machination d'un autre ordre, à côté de laquelle ses inventions ne sont que des petites machinations pour résoudre des problèmes domestiques.

It's a Bird est peut-être, de ce point de vue, l'un des films les plus intrigants de Bowers. Le monde dans lequel $\mathrm{M}$. Bricolo évolue comprend une espèce hybride d'oiseau bien particulière et qu'il ne s'explique pas, un nouvel animal qui s'épanouit aux marges de la civilisation 
industrielle, au fond des garages, des entrepôts et des hangars, très civilisé par la nourriture qu'il absorbe, mais qui reste très sauvage par son comportement et réticent à toute domestication. Au début du film, M. Bricolo est montré travaillant dans une casse de voitures et débordé par l'afflux de ferraille. Manquant d'espace supplémentaire, il se met en quête de l'oiseau mangeur de métal, situé quelque part en Afrique, pour s'en faire un partenaire. Il le capture à l'aide d'un gros ver de terre et le ramène chez lui. L'oiseau se révèle alors capable d'avaler toute la ferraille et de pondre un œuf qui se développe en voiture. M. Bricolo se dit qu'il va pouvoir nouer alliance avec lui et devenir riche, mais l'oiseau le prévient : malheureusement pour lui, un tel miracle ne pourra se produire qu'une fois tous les cent ans! Obsédée par l'intégration de la machine à la nature, l'expérimentation wild tech de Bowers, - où les rapports entre la machine, l'animal et le végétal ne sont pas régis par des lois permettant de passer de l'un à l'autre, mais par des accidents contre-nature, trouve dans cette impossible domestication sa plus belle expression. Bowers ne peut que constater les miracles d'une « nature de synthèse », devenue elle-même un grand garage, qui a fini par aspirer la mécanique en son sein. Ce garage à ciel ouvert improvise à son tour de manière imprévisible et incontrôlable, en toute liberté et parfois à l'insu de ceux qui l'ont alimenté en inventions et procédés de toute sorte. Il génère, de manière quasi darwinienne, des hybrides, dont on n'est jamais sûr de l'intériorité ni du comportement et qui finissent toujours par se retourner contre leurs inventeurs ou contre le milieu dont ils sont éclos. Chez Bowers, inventer ne revient pas simplement à mettre un objet inédit en circulation mais à stimuler et provoquer des puissances jusque-là en sommeil et dont on ne peut prévoir les réactions. Drôle d'exercice de prospective technologique, posant, avec éloquence, ironie et poésie, toute l'ambivalence de l'invention. 


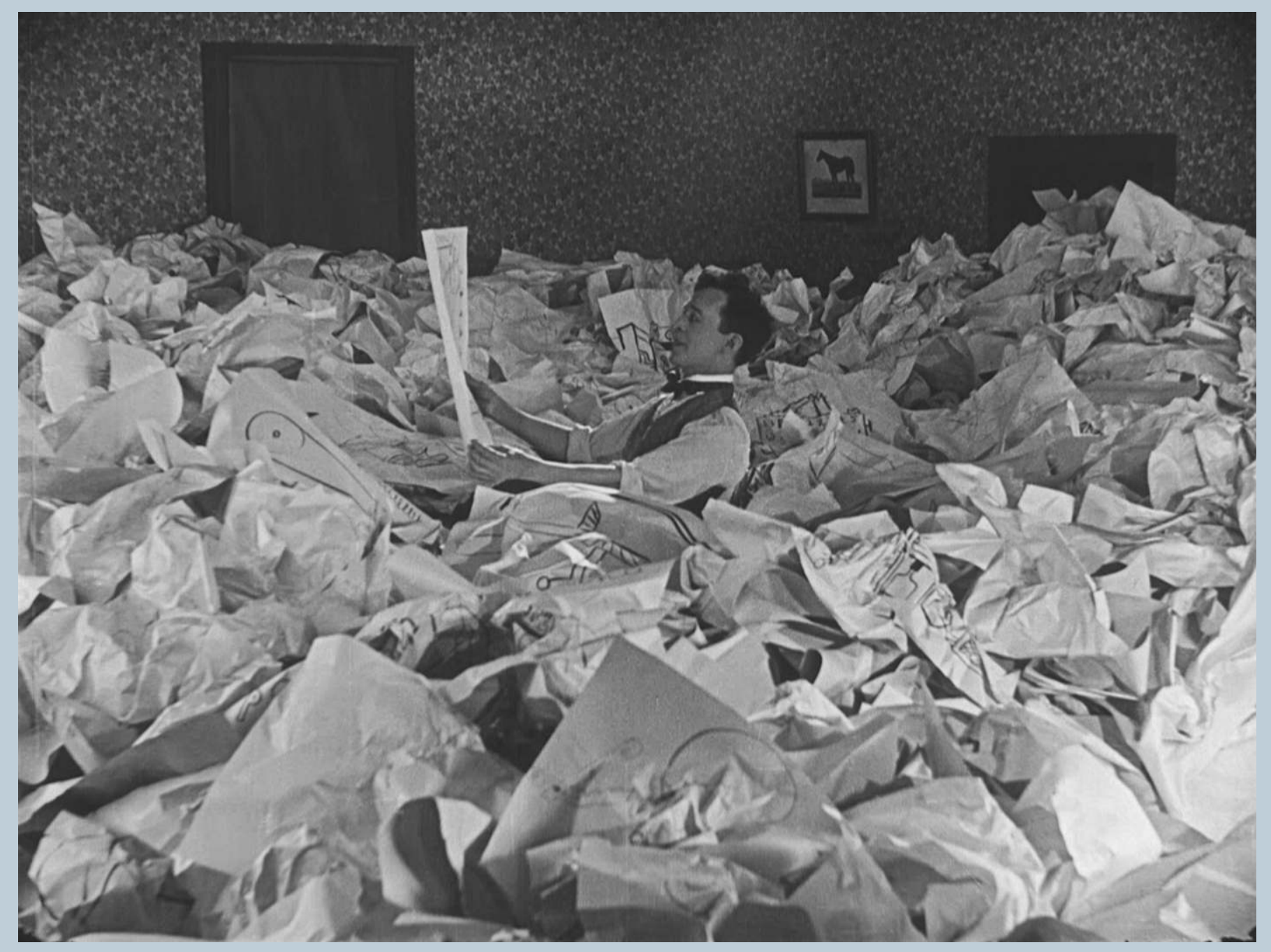




\section{Notes}

1. Dans le champ de l'anthropologie, Pierre Lemonnier (2004) a démontré tout l'intérêt qu'il y a à s'intéresser à des chaînes opératoires mobilisant des matériaux hétérogènes et des connexions renvoyant à d'autres formes de savoir.

2. Pour une discussion de la notion de « circuitousness » dans un volume consacré à la virtuosité technique, voir Stoichita, Grimaud \& Jones (2011).

3. Pour un aperçu des mécanismes alambiqués de Rube Goldberg, dont les premiers datent de 1914, voir Goldberg (2000). Bruno Munari, futuriste italien, auteur d'un Manifeste du machinisme en 1952, a dessiné des mécanismes comparables, réédités ici (Munari 1998). Un très bel exemple de mécanisme «à la Rube Goldberg » a été conçu par Paul Spooner, Tim Hunkin et leurs amis pour le Science Museum de Londres à la fin des années 2000, intitulé On the Move.

4. Pour une interview de Raymond Borde, voir l'intégrale des films de Bowers éditée par Lobster, sous le titre Charley Bowers, The Rediscovery of an American Comic Genius, 2004.

5. Voir sur ce point, la magnifique théorie du lien de Giordano Bruno (1591) et son analyse par Bardini (2007).

6. Voir Lallement 2015 mais aussi toute la littérature consacrée à l'art de stimuler la créativité en design et à la fabrication de «prototypes ».

\section{I'auteur}

Emmanuel Grimaud est anthropologue, chercheur au CNRS (LESC-UMR7186). Ses dernières recherches ont porté sur la robotique et en particulier sur la notion de "vallée de l'étrange ", Robots étrangement humains (avec D. Vidal, Gradhiva 2012), Le Jour où les robots mangeront des pommes (Petra 2012). Il a été commissaire de l'exposition Persona, étrangement humain (avec A C.Taylor, T. Dufrene \& D. Vidal, Actes Sud 2016).

\section{Iconographie}

Image d'ouverture. "Monsieur Bricolo, inventeur », extrait de Many a slip, 1927.

1, 3, 4. Extraits de Now you tell one, 1928.

$2 \& 6$. Extrait de Egged on, 1926.

\section{Références}

Bardini, T. 2007 « Les liens de Giordano Bruno : magie, arts et sciences à l'heure du nexus », Inter, art actuel $97: 20-26$.

Bruno, G., 2001, Des liens. Paris : Allia (édition originale 1591).

Gell, A 1988 « Technology and magic », Anthropology Today 4 (2) : 6-9.

Goldberg, R. 2000 Inventions! New York : Simon and Shuster.

Kawakami, K. 2005 The Big Bento Box Of Unuseless Japanese Inventions: The Art of Chindogu. New York: W. W. Norton \& Company.

- 199799 More Unuseless Japanese Inventions : The Art of Chindogu. New York: W. W. Norton \& Company.
5. Extrait de A wild roomer, 1926

Crédits pour l'ensemble des images. Charley Bowers, un génie à redécouvrir (C) Lobster Films.

— 1995101 Unuseless Japanese Inventions. New York: W. W. Norton \& Company.

Lallement, M. 2015 L'âge du faire. Hacking, travail, anarchie. Paris : Seuil.

Lemonnier, P. 2004 « Mythiques chaînes opératoires », Techniques\&Culture 43-44. En ligne: tc.revues. org/1054.

Mauss, M. \& Hubert H. 1902 Esquisse d'une théorie générale de la magie, L'Année sociologique 1902-1903:41.

Meyer, M. 2012 « Bricoler, domestiquer et contourner la science : l'essor de la biologie de garage ", Réseaux 173-174: 303-328.

Munari, B. 1998 Les machines de Munari. Queyrac : Éditions Delphine Montaland. 
Solomon, S. 2010 « Slapstick Modernism. Charley Bowers and Industrial Modernity », Modernist Cultures 2 (2) : 170-188. DOI : http://dx.doi.org/10.3366/ E2041102209000264.

Spooner, P., Hunkin, T. et al. 2007. On the Move. Science Museum of London : www.sciencemuseum.org.uk/ videos/onthemove.aspx.
Stoichita, V., Grimaud, E. \& G. Jones 2011 « De la technique comme contorsion», in Virtuosité ou les sublimes aventures de la technique, Ateliers d'Anthropologie 35. En ligne : ateliers.revues.org/8838.

\section{Filmographie}

Charley Bowers, The Rediscovery of an American Comic Genius, Lobster Films, 2004.

\section{Pour citer l'article}

Grimaud, E. 2017 «Wild Tech. Repenser l'invention avec Charlie Bowers », Techniques\& Culture 67 « Low tech? Wild tech! », p. 258-279. 